\title{
Input-Dependent Integral Nonlinearity Modeling for Pipelined Analog-Digital Converters
}

\author{
Samer Medawar, Peter Händel, Senior Member, IEEE, Niclas Björsell, Member, IEEE, and \\ Magnus Jansson, Member, IEEE
}

\begin{abstract}
Integral nonlinearity (INL) for pipelined analogdigital converters (ADCs) operating at RF is measured and characterized. A parametric model for the INL of pipelined ADCs is proposed, and the corresponding least-squares problem is formulated and solved. The INL is modeled both with respect to the converter output code and the frequency stimuli, which is dynamic modeling. The INL model contains a static and a dynamic part. The former comprises two 1-D terms in ADC code that are a sequence of zero-centered linear segments and a polynomial term. The 2-D dynamic part consists of a set of polynomials whose parameters are dependent on the ADC input stimuli. The INL modeling methodology is applied to simulated and experimental data from a 12-bit commercial ADC running at 210 mega samples per second. It is demonstrated that the developed methodology is an efficient way to capture the INL of nowadays ADCs running at RF, and it is believed that the methodology is powerful for INL-based ADC postcorrection in wideband applications.
\end{abstract}

Index Terms-Analog-digital conversion, integral nonlinearity (INL), least-squares methods, parametric modeling, postcorrection, segmentation.

\section{INTRODUCTION}

$\mathbf{T}$ $\mathrm{HE}$ analog-digital converter (ADC) is a pervasive component in any digital communication system. The role of an ADC working at RF is becoming more crucial with new wideband wireless communications systems, including long-term evolution (LTE) and worldwide interoperability for microwave access (Wimax). For example, the LTE system supports bandwidths up to $20 \mathrm{MHz}$. Upcoming technologies are expected to sustain even larger bandwidths. An ADC operating at RF encounters different input signal scenarios in terms of frequency span, time-domain characteristics, and spread in signal power levels. The ADC input-output linearity over the whole bandwidth is important to ensure consistent performance.

\section{A. Pipelined ADCs}

There is a tradeoff between the ADC speed of conversion and its resolution. As the sampling speed of the ADCs in-

Manuscript received June 17, 2009; revised November 26, 2009. This work was supported by the European Research Council under the European Community's Seventh Framework Program (FP7/2007-2013)/ERC Grant 228044. The Associate Editor coordinating the review process for this paper was Dr. Wendy Van Moer.

S. Medawar, P. Händel, and M. Jansson are with the Signal Processing Laboratory, ACCESS Linnaeus Center, Royal Institute of Technology, 10044 Stockholm, Sweden.

N. Björsell is with the Center for RF Measurement Technology, University of Gävle, 80176 Gävle, Sweden.

Digital Object Identifier 10.1109/TIM.2010.2045551 creases, their output resolution decreases. The ADC internal hardware structure has a direct impact on its working range. The successive approximation ADC operational scheme consists mainly of repeatedly comparing the input voltage to internal digitized levels until a best approximation is achieved. It has a sampling frequency in the range of $1-10 \mathrm{MHz}$, with a resolution between 8 and 16 bits. The pipeline or subranging ADC can be considered to be a refined version of the successive approximation ADC. Thus, it has the same or slightly higher resolution, whereas its sampling rate ranges from $10 \mathrm{MHz}$ to $2.5 \mathrm{GHz}$ [1], [2].

The structure of a conventional pipelined ADC is shown in Fig. 1 [3]. The analog input $s$ is fed to the subranging ADC, where the signal goes through the subsequent $S$ pipelined stages and the final Flash ADC. Each pipeline ADC stage consists mainly of a Flash ADC, a sample-and-hold entity, a digital-analog converter (DAC), and a stage amplifier, where the latter three blocks contribute to the error generated in each stage and the given ADC in general. In the first stage, the analog voltage is quantized by an $N_{1}$-bit Flash ADC, and the obtained code is directed to the output code register. The obtained code is used to address an $N_{1}$-bit DAC. The output of the DAC is subtracted from the sampled analog input voltage, and the residual is amplified by gain $g$ (as shown for stage 2 in this presentation) prior to being fed to the next stage. The process continues through the remaining $S-1$ stages, where each provides $N_{1}$ additional bits to the output code register. The gained residual of stage $S$ is fed into an $N_{2}$-bit Flash ADC that resolves the remaining output code of the ADC. The $N$-bit output code $k$ is constructed with the individual $\left(S \times N_{1}+N_{2}\right)$ subcodes provided by the $S$ stages and the Flash ADC. With regard to timing, each stage resolves the subsequent signal sample after each clock cycle, which explains the high throughput of the pipeline structure. However, due to the pipelined ADC structure, each stage contributes to the quantization error in a certain input range or, equivalently, in the output code $k$ interval. The sample-and-hold block is basically composed of a capacitor and a switch diode. The capacitor endures hysteresis effects due to the nonidentical charging and discharging phenomena, which causes the sampled quantity to be different from the input signal. With regard to the DAC, the embedded capacitor mismatch affects its gain and binary weights [4]. The stage amplifier can hardly be optimized to simultaneously have a short settling time and an accurate gain [4]. Additional error sources include the amplifier's gain bandwidth product, the finite dc gain, interstage reference mismatch, and clock jitter [5]. These errors translate into faulty transition 


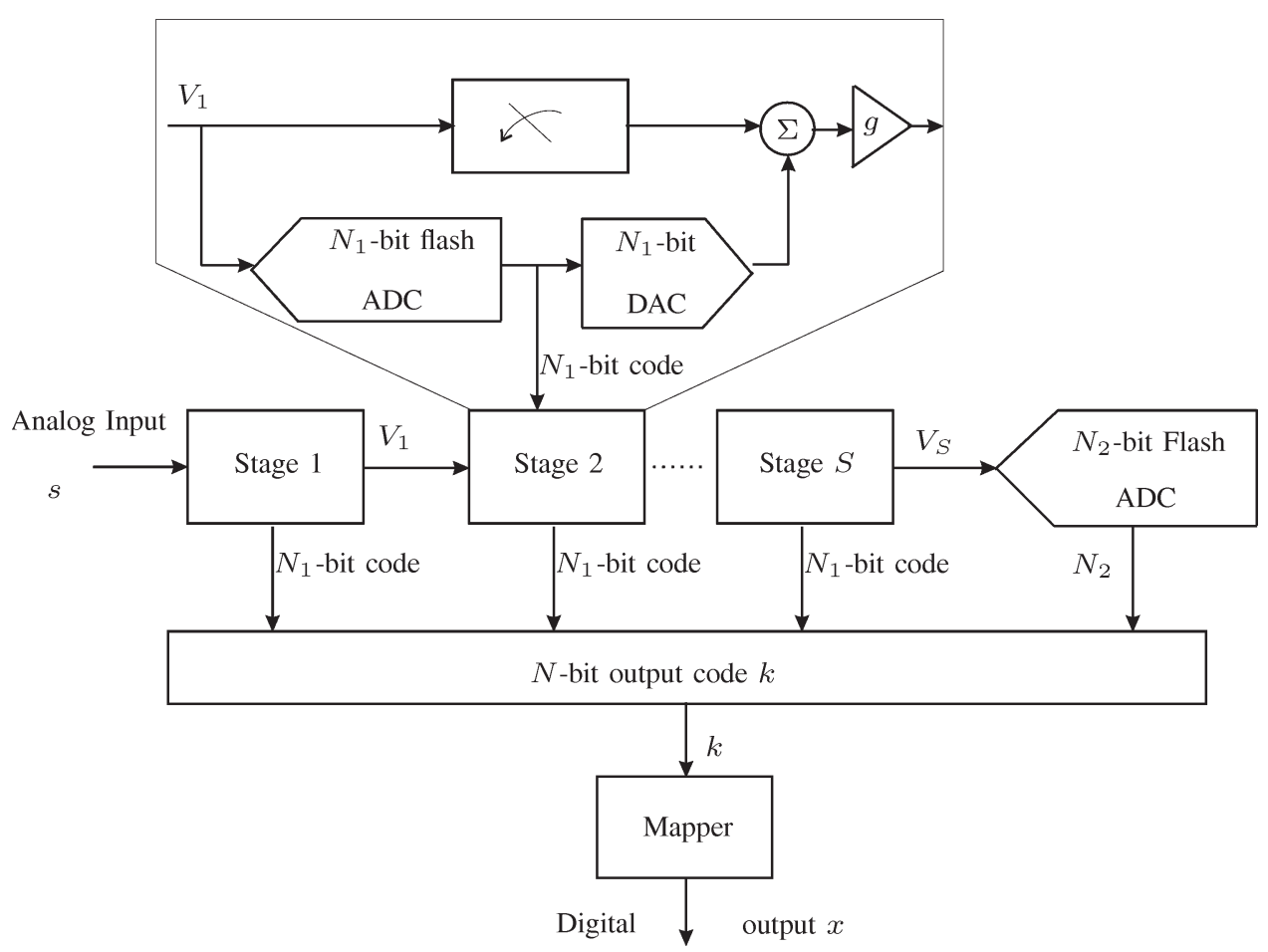

Fig. 1. Static description of a pipeline ADC, where the input $s$ produces the digital output $x$ through the output code $k$.

levels that deviate from the ideal ones, in addition to a gain and offset compared with an ideal transfer curve.

\section{B. Static and Dynamic ADC INL}

The ADC circuitry affects the ADC figure of merits, such as integral nonlinearity (INL). The INL is the measure of practical ADC deviations compared with an ideal staircase quantizer [2], [6]. The code transition levels $T_{k}$ (in volts) of an ideal ADC can be related to the output code $k$ of the ADC by [7, Sec. 4.3.1]

$$
T_{k}=Q \cdot(k-1)+T_{1} \quad \text { (in volts) }
$$

where $Q$ (in volts) is the ideal width of a code bin, which is the full-scale range of the ADC divided by the total number of codes $\left(V_{\max }-V_{\min }\right) / 2^{N} \cdot T_{1}$ is the first ideal transition level, and its value is equal to $V_{\min }+Q$ for the "midriser" convention or to $V_{\min }+Q / 2$ for the "midtread" [7, Sec. 4.3.1]. Because a practical ADC is not ideal, the actual transition levels are designated by $T[k]$ (in volts), as illustrated in Fig. 2. In addition, the output of a real-life ADC suffers certain gain and offset errors compared with the ideal staircase function. The INL is defined as the difference between $T_{k}$ given in (1) and $T[k]$, after correcting for the gain and offset of the transfer curve of the ADC [7]. First, the residual is given by $\varepsilon[k]=T_{k}-G \cdot T[k]-V_{\text {os }}$ (in volts), where $G$ and $V_{\text {os }}$ are the gain factor and offset term, respectively. The gain and offset can be found using the least-squares method or, alternatively, by enforcing a null residual at the terminal codes.

The INL is normally expressed in LSBs, where an LSB is synonymous with one ideal code bin width $Q$ (in volts); that is, $i[k]=\varepsilon[k] / Q$ (in LSBs), where $i[k]$ denotes the INL as a

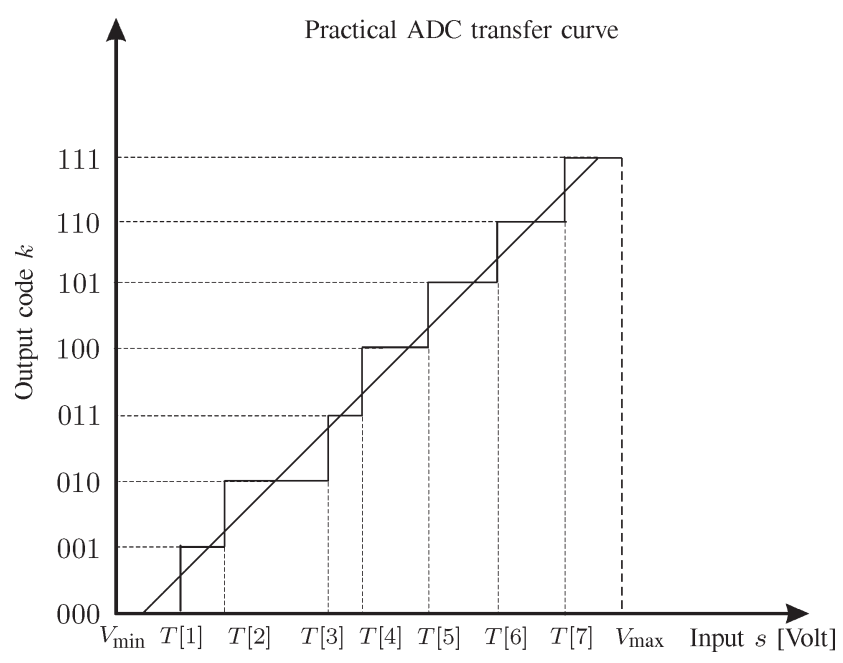

Fig. 2. Practical 3-bit midriser ADC transfer curve, compensated for gain and offset errors.

function of $k$. A typical INL sequence measured for a 12-bit pipelined ADC (AD9430) is shown in Fig. 3.

In a first approximation, the INL is depicted as a 1-D sequence in terms of the ADC output code $k$, as displayed in Fig. 3. As observed from the figure, the pipelined structure causes abrupt variations in consecutive output codes, thus producing an INL with almost linear behavior. The sharp sawtooth INL behavior is often denoted as the high-code frequency (HCF) component in the literature [8]-[10]. Another type of error common to RF devices is harmonic distortion generated by the nonlinear components. This harmonic distortion is reflected in the INL by a polynomial-shaped variation in the output code domain; its coefficients are related to the harmonics amplitudes or levels [11]. The polynomial behavior of the INL is clearly 


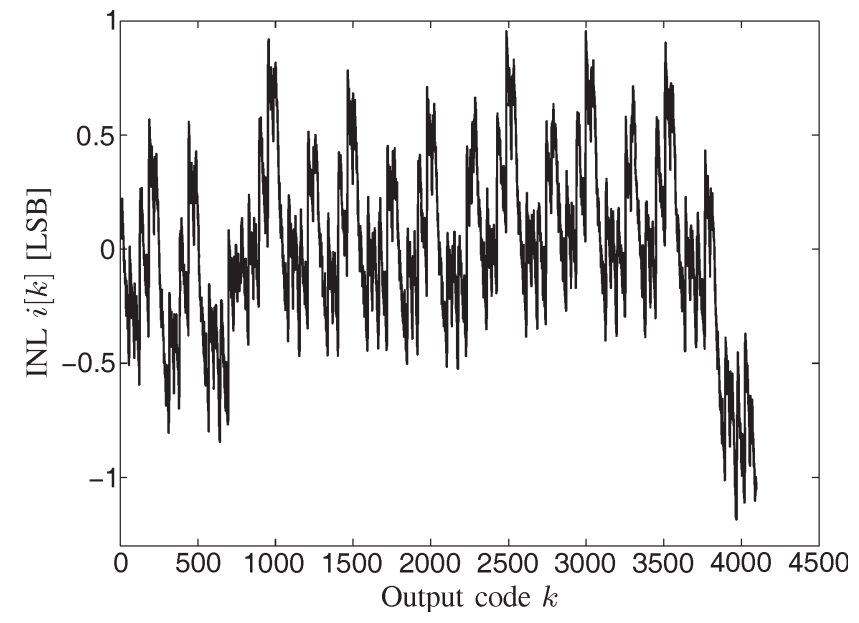

Fig. 3. Average INL for 15 sequences corresponding to test frequencies in the interval 30-90 MHz for a 12-bit pipelined ADC (AD9430).

visible in the sample data in Fig. 3. This INL behavior is known as the low-code frequency (LCF) component [12].

In a more flexible model of ADC imperfections, the INL is dependent on the input signal characteristics, such as input frequency, slope, or phase. This INL behavior is regarded as dynamic because it is the outcome of certain input parameters or exists for specified input conditions. In other words, the output code $k$ could be different for two input signals that have identical instantaneous amplitudes but different frequencies or slopes. Thus, the INL model is often considered to have two degrees of freedom: one directly coupled to the output code and one that reflects the dynamic behavior, such as frequency or slope.

\section{State of the Art}

A dynamic INL model consisting of one term was developed in [13]. The model was represented by a single 2-D polynomial, where the slope of the signal was added to the code dimension to represent the INL, i.e., a phase-plane representation. The main rationale behind this approach was to take into account the hysteresis behavior inherent to the converter, whose knowledge was used in postcorrection.

The INL model presented in [12] consisted of two static terms: the LCF was modeled by a low-order polynomial, whereas the HCF was related to the multiperiodic occurrence of the differential nonlinearity (DNL). Basically, the HCF was computed as the cumulative sum with respect to $k$ of the DNLs. For a model similar to that employed in [12], a triangular signal at the ADC input was used to identify the INL model parameters in [8]. The histogram test with a sinusoidal stimulus was used to estimate the HCF in [10]. In [14] and [15], a triangular stimulus was used for the narrow-band histogram test (i.e., using a triangular signal with a very smooth or reduced slope that translates into a narrow-band signal in the frequency domain) of the HCF, and multiharmonic sine fitting in the time domain was performed to estimate the LCF.

A dynamic INL model composed of two terms was proposed in [16]. The HCF term was modeled by static piecewiselinear segments, whereas the LCF was determined as a dy- namic polynomial that depends on the frequency of the input stimuli. The model was investigated and parameterized in [17]. The parameters were estimated using least-squares fit. The method was applied on simulated data, and the proposed model structure was effective in describing the INL data structure. Modifications of the method in [17], where experimental data were also employed, were made in [18].

\section{Motivation and Contributions}

The main purpose of this paper is to develop and investigate a parametric INL model that mimics the INL information of nowadays pipelined ADCs running at RF. The model captures the behavior of the INL with respect to the output code $k$, as well as the dependence of the INL on the frequency content of the ADC test stimuli. Thus, the model is dynamic in the sense that it mimics the INL of the ADC both with respect to the output code $k$ and the frequency content of the ADC input. The studies in [16]-[18] implicitly reveal that the two-term INL model can be refined into a model with three main components: 1) a set of piecewise-linear (linear in code $k$ ) segments; 2) a 1-D polynomial in code $k$; and 3) a 2-D polynomial in $k$ and $m$, where $m$ is an integer index related to the frequency content of the ADC input stimuli [19]. This refined three-term model is studied in this paper. This work considers the pipeline structure because it is the typical ADC of a nowadays radio base station for wireless communications. The developed parametric error model mimics the behavior of the ADC pipeline structure, but we emphasize that the methodology as such can be applied to any ADC structure, as long as the INL behavior can suitably be parameterized. The key issue is to find a parametric model (preferably linear in the parameters) that is sufficiently accurate and flexible. In our work, the 12-bit 210 mega samples per second (MSPS) AD9430 by Analog Devices is used to experimentally verify the theoretical results.

This paper is organized as follows: Section II presents parametric INL modeling, where the estimation problem is formulated. The problem is then solved in Section III, subject to a given model structure. The problem of model order determination and data segmentation is addressed in Section IV. Section V presents the experimental study, employing both simulated and measured data. This paper is concluded in Section VI.

\section{INL MODELING}

The aim of this section is to provide a parametric description of the INL data subject to the characteristics of the ADC input stimuli.

\section{A. Representation of the Measured INL}

To characterize the INL, a plurality of sine-wave tests is used. The frequencies $\left\{f_{m}\right\}_{m=1}^{M}$ (in hertz) of the input sine wave span the band of interest. Thus, the sine-wave test stimuli generate an INL sequence for each test frequency. Frequency stepping is performed over the bandwidth of interest; thus, a finite number $M$ of INL sequences is obtained. The resulting INL is denoted by $i[m, k]$, where $k$ denotes the code and $m$ is an integer that 


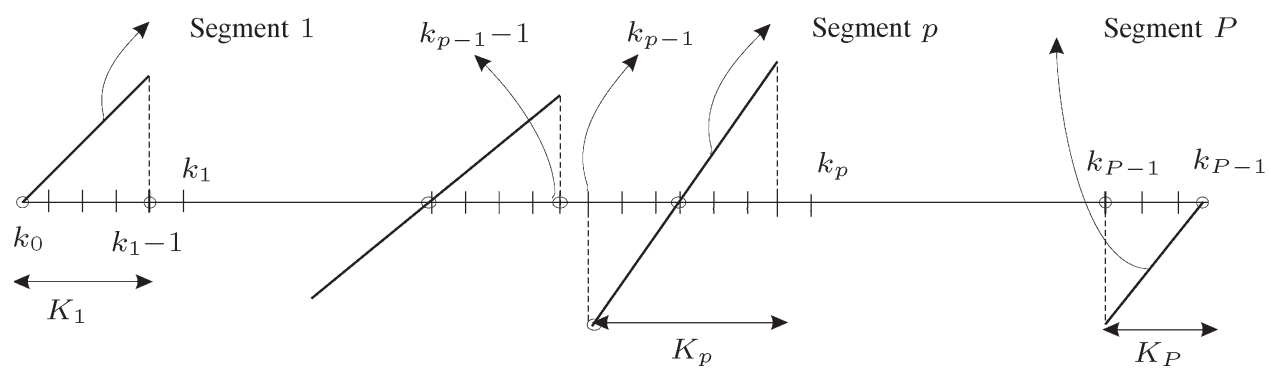

Fig. 4. Centered $P$ segment scheme, where $k_{0}=1$ and $k_{P}-1=2^{N}-1$. Note that the first and the $P$ th segments are forced to zero at the corresponding start and end points, respectively.

represents the frequency. Thus, for a given input stimulus, of frequency $f_{m}$, the INL record collected in a column vector is given by

$$
\mathbf{i}[m]=\left(\begin{array}{c}
i[m, 1] \\
\vdots \\
i\left[m, 2^{N}-1\right]
\end{array}\right), \quad m=1, \ldots, M .
$$

Thus, $\mathbf{i}[m]$ represents the measurement data corresponding to the stimulus frequency $f_{m}$ (in hertz). To employ a compact notation, the $M$ measured INL sequences are stacked in the augmented vector $\mathbf{i}$, which is given by

$$
\mathbf{i}=\left(\begin{array}{c}
\mathbf{i}[1] \\
\vdots \\
\mathbf{i}[M]
\end{array}\right)
$$

In (3), $\mathbf{i}$ is a column vector with $\left(2^{N}-1\right) M$ entries. In the following, a parametric model for the measured INL is introduced. The aim is to derive a model that reflects the behavior of the INL with respect to both the code $k$ and the input frequency $f_{m}$.

Let $i_{m, k}$ denote the INL model output corresponding to the measurement $i[m, k]$ for a given $m$ and $k$. Then, the INL model in terms of static HCF and dynamic LCF can be expressed, for $m=1, \ldots, M$ and $k=1, \ldots, 2^{N}-1$, as

$$
i_{m, k}=h_{k}+\ell_{m, k}
$$

where $h_{k}$ is the static HCF component, and $\ell_{m, k}$ is the LCF component. A piecewise-linear model is employed for the static HCF given by $h_{k}$. The LCF is modeled by a polynomial in $k$ with frequency-dependent coefficients. In [18], the $\ell_{m, k}$ polynomials were shown to have common features that do not change with frequency. Thus, a static term $s_{k}$ embedded in the LCF component is obtained by taking the arithmetic mean of the polynomial data with respect to the frequency, i.e.,

$$
s_{k} \triangleq \frac{1}{M} \sum_{m=1}^{M} \ell_{m, k}
$$

By construction, the dynamic LCF $d_{m, k}$ becomes

$$
d_{m, k} \triangleq \ell_{m, k}-s_{k} .
$$

The representation (4) of the INL model is now refined as

$$
i_{m, k}=\underbrace{h_{k}+s_{k}}_{\text {static part }}+\underbrace{d_{m, k}}_{\text {dynamic part }} .
$$

In summary, the model $i_{m, k}$ of the measured INL $i[m, k]$ consists of two input-independent terms (the $\mathrm{HCF} h_{k}$ and the static part $s_{k}$ of the LCF $\ell_{m, k}$, respectively) and the dynamic part of the LCF, which is denoted $d_{m, k}$. The latter is a term that depends on both the code $k$ and the frequency $f_{m}$. Furthermore, $s_{k}$ is a continuous function of $k$ with no abrupt changes, as is $h_{k}$, which is composed by isolated linear segments.

\section{B. HCF Modeling}

The HCF components $h_{k}$ in (7) originates from the ADC circuitry imperfections in the pipelined stages. The dominant error occurs in the DACs, which converts the $N_{1}$-bit code received from the Flash ADC. This is a frequency-independent phenomenon, because the DAC receives a specified $N_{1}$-bit input code every clock cycle. Thus, the input code is converted independently of the analog input signal $s$ dynamics. Errors are due to the DAC inherent switched-capacitor circuit, which is directly affected by the value of the input code [4]. As a result, the INL undergoes roughly linear changes in consecutive code $k$ intervals. This behavior is reflected by the static HCF component. On the other hand, the embedded Flash converters do not contribute to the INL since they are used to convert the signal at a lower resolution (i.e., at $N_{1}$ bits) than their capability. In other words, the individual Flash ADCs convert the signal at a resolution that is higher than $N_{1}$, but the structure is satisfied with the $N_{1}$ resolution and forwards the residual signal to the next stage in the structure. Thus, it can be considered to be error free; that is, it does not contribute to the INL.

The high-code INL data $h_{k}$ are modeled by $P$ piecewiselinear segments centered around zero, as illustrated in Fig. 4. The HCF in a specific interval $K_{p}$ is modeled by

$$
h_{k} \triangleq h_{k, p}=\left(k-\frac{k_{p-1}+k_{p}-1}{2}\right) \eta_{p}
$$

where $p$ refers to the ordered code intervals ranging from 1 to $P$. That is

$$
K_{p}:\left\{k \mid k_{p-1} \leq k<k_{p}\right\} .
$$

The output code has as lower and upper endpoints, i.e., $k_{0}=1$ and $k_{P}=2^{N}$, respectively. The set of regressor coefficients $\eta_{p}$ is gathered in a vector $\boldsymbol{\eta}$ of size $P$, i.e.,

$$
\boldsymbol{\eta}=\left(\eta_{1}, \ldots, \eta_{p}\right)^{T}
$$


where $T$ denotes transpose. Each component in $\boldsymbol{\eta}$ represents the slope of the individual segments.

Employing vector notation, the HCF is written as $\mathbf{h} \boldsymbol{\eta}$, where $\mathbf{h}$ is a $\left(2^{N}-1\right) \times P$ block diagonal matrix defined by a set of column vectors $\mathbf{h}_{1}, \ldots, \mathbf{h}_{P}$ [defined in (12)], i.e.,

$$
\mathbf{h}=\left(\begin{array}{ccc}
\mathbf{h}_{1} & 0 & \\
0 & \ddots & 0 \\
& 0 & \mathbf{h}_{p}
\end{array}\right) .
$$

In (11), an arbitrary column $p$ is formed by the column vector $\mathbf{h}_{p}$ with a size equal to the length of the $p$ th segment (e.g., $\bar{p}_{p}$ ) and an additional $2^{N}-1-\bar{p}_{p}$ zeros. A given segmentation of the INL data determines the actual form of the introduced vectors, i.e., $\mathbf{h}_{p}$ in (11), which depend on the number and size of the segments (9). The HCF segmentation procedure based on the measured INL is nontrivial and studied in detail in Section IV. For $1<p<P$, it holds that the vectors $\mathbf{h}_{p}$ in (11) are given by

$$
\mathbf{h}_{p}=\left(\begin{array}{c}
-\frac{k_{p}-k_{p-1}-1}{2} \\
\vdots \\
\frac{k_{p}-k_{p-1}-1}{2}
\end{array}\right)
$$

The first point of the segment $p=1$ and the last point of the segment $p=P$ are set to zero. Accordingly, $\mathbf{h}_{1}$ and $\mathbf{h}_{P}$ are given by

$$
\mathbf{h}_{1}=\left(\begin{array}{c}
0 \\
1 \\
\vdots \\
k_{1}-2
\end{array}\right) \quad \mathbf{h}_{p}=\left(\begin{array}{c}
k_{P-1}-\left(k_{p}-1\right) \\
\vdots \\
1 \\
0
\end{array}\right) .
$$

\section{LCF Modeling}

The LCF represents a smooth and continuous change of the INL over the whole code range. The dynamic and static components constituting the LCF [i.e., $\ell_{m, k}$ in (4)] are modeled by a polynomial of order $L$. For large values of $N$, polynomial modeling is sensitive to rounding errors. Recall that HCF modeling is performed with the origin centered within each segment $p$, as given by (8). Similarly, LCF modeling employs a normalized (over the whole code range $2^{N}-1$ ) code $\bar{k}$ that spans the interval -1 to 1 . Accordingly, for LCF modeling

$$
\bar{k}=\frac{k-2^{N-1}}{2^{N-1}-1} .
$$

Therefore, the polynomial part $\ell_{m, k}$ in (4) can be written as an $L$ th-order polynomial in $\bar{k}$, i.e.,

$$
\ell_{m, k}=\theta_{0, m}+\theta_{1, m} \bar{k}+\cdots+\theta_{L, m} \bar{k}^{L}
$$

where the dependence of the polynomial on the ADC stimuli frequency $f_{m}$ is indicated by the subscript $m$. In short, using (15)

$$
\ell_{m, k}=\boldsymbol{\ell}_{k}^{T} \boldsymbol{\theta}_{m}
$$

where $\boldsymbol{\theta}_{m}$ is a frequency-dependent parameter vector of length $L+1$, i.e.,

$$
\boldsymbol{\theta}_{m}=\left(\theta_{0, m}, \ldots, \theta_{L, m}\right)^{T}
$$

and $\ell_{k}$ is the regressor with the normalized code $\bar{k}$ values, i.e.,

$$
\boldsymbol{\ell}_{k}=\left(\begin{array}{c}
1 \\
\frac{k-2^{N-1}}{2^{N-1}-1} \\
\vdots \\
\left(\frac{k-2^{N-1}}{2^{N-1}-1}\right)^{L}
\end{array}\right)
$$

Once again, employing vector notation, the LCF sequence for a given frequency can be modeled by the vector

$$
\ell_{m}=\ell \boldsymbol{\theta}_{m}
$$

where the matrix $\ell$ is formed from the regressors $\ell_{1}, \ldots, \ell_{2^{N}-1}$. The Vandermonde $\left(2^{N}-1\right) \times(L+1)$-matrix $\ell$ is given by

$$
\ell=\left(\begin{array}{cccc}
1 & -1 & \cdots & (-1)^{L} \\
\vdots & \vdots & & \vdots \\
1 & \frac{k-2^{N-1}}{2^{N-1}-1} & \cdots & \left(\frac{k-2^{N-1}}{2^{N-1}-1}\right)^{L} \\
\vdots & \vdots & & \vdots \\
1 & 1 & \cdots & 1^{L}
\end{array}\right)
$$

The polynomial order $L$ is a design variable, and its selection is discussed in Section IV. Roughly, it should be large enough to characterize the polynomial attributes inherent in the INL data.

\section{LEAST-SQuARES EStimation OF INL PARAMETERS}

From the previous section, we may now gather the parametric models to characterize the measured INL. Recall that an INL characterization corresponding to a test stimulus at $f_{m}$ (in hertz) results in $\mathbf{i}[m]$, as introduced in (2), for $m=1, \ldots, M$. The least-squares problem is formulated in the following discussion, subject to a given LCF polynomial order $L$, and a given HCF segmentation defined in (9).

\section{A. INL Vector Model}

Let $m$ represent a test frequency $f_{m}$ (in hertz) for which the INL data are gathered in vector $\mathbf{i}[m]$, as given by (2). Similarly, the parametric INL data model for a certain frequency $f_{m}$ can be gathered in a vector $\mathbf{i}_{m}$, which is given by

$$
\mathbf{i}_{m}=\left(\begin{array}{c}
i_{m, 1} \\
\vdots \\
i_{m, 2^{N}-1}
\end{array}\right), \quad m=1, \ldots, M .
$$

The measured INL at $f_{m}$ can be described as

$$
\mathbf{i}[m]=\mathbf{i}_{m}+\mathbf{e}_{m}
$$

where $\mathbf{e}_{m}=\left(e_{m, 1}, \ldots, e_{m, 2^{N}-1}\right)^{T}$ is introduced as a zeromean noise term that gathers model imperfections and measurement noise. Furthermore, the INL model comprises the HCF and LCF terms, i.e.,

$$
\mathbf{i}_{m}=\mathbf{h} \boldsymbol{\eta}+\ell \boldsymbol{\theta}_{m}
$$


where the known regressor $\mathbf{h}$ was defined in (11), the HCF parameter vector $\boldsymbol{\eta}$ was defined in (10), the known Vandermonde matrix was defined in (20), and the frequency-dependent LCF parameter vector $\boldsymbol{\theta}_{m}$ was defined in (17).

\section{B. Parameter Estimation by Least Squares}

Since the input frequency has $M$ steps over the whole bandwidth of interest, the multiple experiment data model yields

$$
\underbrace{\left(\begin{array}{c}
\mathbf{i}[1] \\
\vdots \\
\mathbf{i}[M]
\end{array}\right)}_{\mathbf{i}}=\underbrace{\left(\begin{array}{cccc}
\mathbf{h} & \boldsymbol{\ell} & & \\
\vdots & & \ddots & \\
\mathbf{h} & & \boldsymbol{\ell}
\end{array}\right)}_{\left[\begin{array}{ll}
\mathbf{H} & \mathbf{L}]
\end{array}\right]} \underbrace{\left(\begin{array}{c}
\boldsymbol{\eta} \\
\boldsymbol{\theta}_{1} \\
\vdots \\
\boldsymbol{\theta}_{M}
\end{array}\right)}_{\left[\begin{array}{c}
\boldsymbol{\eta} \\
\boldsymbol{\Theta}
\end{array}\right]}+\underbrace{\left(\begin{array}{c}
\mathbf{e}_{1} \\
\vdots \\
\mathbf{e}_{M}
\end{array}\right)}_{\mathbf{e}} .
$$

The matrices $\mathbf{H}, \mathbf{L}, \Theta$, and $\mathbf{e}$ are implicitly defined by (24). To summarize, the INL data set for each test frequency $m$ is represented by static piecewise-linear segment components $\mathbf{h} \eta$ (the term is the same for all experiments that provide INL data, i.e., independent of $\mathrm{ADC}$ frequency stimulus) and a polynomial term $\boldsymbol{\ell} \boldsymbol{\theta}_{m}$, with frequency-dependent parameters in the vectors $\left\{\boldsymbol{\theta}_{m}\right\}_{m=1}^{M}$.

The solution to (24) can be derived by exploiting the inherent structure. Thus, starting with the compact notation for (24)

$$
\mathbf{i}=\left[\begin{array}{ll}
\mathbf{H} & \mathbf{L}
\end{array}\right]\left[\begin{array}{l}
\boldsymbol{\eta} \\
\Theta
\end{array}\right]+\mathbf{e} .
$$

The least-squares solution is

$$
\left[\begin{array}{c}
\widehat{\boldsymbol{\eta}} \\
\widehat{\mathbf{\Theta}}
\end{array}\right]=\left[\begin{array}{ll}
\mathbf{H} & \mathbf{L}
\end{array}\right]_{\mathbf{I}}^{\dagger} \mathbf{i}=\left(\left[\begin{array}{l}
\mathbf{H}^{T} \\
\mathbf{L}^{T}
\end{array}\right]\left[\begin{array}{ll}
\mathbf{H} & \mathbf{L}
\end{array}\right]\right)^{-1}\left[\begin{array}{l}
\mathbf{H}^{T} \\
\mathbf{L}^{T}
\end{array}\right] \mathbf{i}
$$

where the ${ }^{-}$is intended to represent estimated quantities or parameters. The notation $\mathbf{X}_{\mathbf{Q}}^{\dagger}$ for the $\mathbf{Q}$-weighted pseudoinverse of a matrix $\mathbf{X}$ is introduced as

$$
\mathbf{X}_{\mathbf{Q}}^{\dagger}=\left(\mathbf{X}^{T} \mathbf{Q X}\right)^{-1} \mathbf{X}^{T} \mathbf{Q} .
$$

In (26), an unweighted pseudoinverse is used, such that $\mathbf{Q}=\mathbf{I}$, where $\mathbf{I}$ denotes the identity matrix with the proper dimensions. Let $\Pi_{\perp}$ be the projection matrix

$$
\boldsymbol{\Pi}_{\perp}=\mathbf{I}-\mathbf{L}\left(\mathbf{L}^{T} \mathbf{L}\right)^{-1} \mathbf{L}^{T} .
$$

Then, one can show that the least-squares solution (26) can be separated into one solution for $\boldsymbol{\eta}$ and one coupled solution for $\Theta[17]$, i.e.,

$$
\begin{aligned}
\widehat{\boldsymbol{\eta}} & =\mathbf{H}_{\Pi_{\perp}}^{\dagger} \mathbf{i} \\
\widehat{\boldsymbol{\Theta}} & =\mathbf{L}_{\mathbf{I}}^{\dagger}(\mathbf{i}-\mathbf{H} \widehat{\boldsymbol{\eta}}) .
\end{aligned}
$$

Exploiting the structures inherent in $\mathbf{L}$, the quantity $\mathbf{L}^{T} \mathbf{L}$ is found to be block diagonal with a block diagonal inverse. Accordingly, the square matrix $\Pi_{\perp}$ in (28) is block diagonal, i.e.,

$$
\boldsymbol{\Pi}_{\perp}=\operatorname{blockdiag}\left(\boldsymbol{\pi}_{\perp}, \ldots, \boldsymbol{\pi}_{\perp}\right)
$$

where $\pi_{\perp}$ is square of size $2^{N}-1$, which is given by

$$
\boldsymbol{\pi}_{\perp}=\mathbf{I}-\ell\left(\ell^{T} \ell\right)^{-1} \ell^{T} .
$$

It is worth noting that the inverse of the $(L+1)$ square matrix $\left(\ell^{T} \ell\right)$ is needed. Making use of the block structure of the matrix $\mathbf{H}=\left[\mathbf{h}^{T}, \ldots, \mathbf{h}^{T}\right]^{T}$, the following equality is obtained:

$$
\mathbf{H}^{T} \boldsymbol{\Pi}_{\perp} \mathbf{H}=M \mathbf{h}^{T} \boldsymbol{\pi}_{\perp} \mathbf{h}
$$

where the fact that $\Pi_{\perp}$ is block diagonal is used. Similarly

$$
\mathbf{H}^{T} \boldsymbol{\Pi}_{\perp} \mathbf{i}=\sum_{m=1}^{M} \mathbf{h}^{T} \boldsymbol{\pi}_{\perp} \mathbf{i}[m] .
$$

In the following discussion, the aforementioned decoupled solutions are further refined. Employing the finding in (34) in combination with the inverse of (33), the least-squares solution (29) is simplified to

$$
\widehat{\boldsymbol{\eta}}=\frac{1}{M}\left(\mathbf{h}^{T} \boldsymbol{\pi}_{\perp} \mathbf{h}\right)^{-1} \mathbf{h}^{T} \boldsymbol{\pi}_{\perp} \sum_{m=1}^{M} \mathbf{i}[m]=\mathbf{h}_{\boldsymbol{\pi}_{\perp}}^{\dagger} \overline{\mathbf{i}}
$$

where $\mathbf{h}_{\boldsymbol{\pi}_{\perp}}^{\dagger}$ is a $P \times\left(2^{N}-1\right)$ weighted pseudoinverse of $\mathbf{h}$, and $\overline{\mathbf{i}}$ is the arithmetic average of the $M$ measured INL sets, i.e.,

$$
\overline{\mathbf{i}}=\frac{1}{M} \sum_{m=1}^{M} \mathbf{i}[m]
$$

To simplify (30), we note that $\mathbf{L}_{\mathbf{I}}^{\dagger}$ is block diagonal with elements $\left(\ell^{T} \ell\right)^{-1} \ell^{T}$. Accordingly, the solution of the $M$ separate vectors $\boldsymbol{\theta}_{m}$ of $\Theta$ can be derived as

$$
\widehat{\boldsymbol{\theta}}_{m}=\left(\boldsymbol{\ell}^{T} \boldsymbol{\ell}\right)^{-1} \boldsymbol{\ell}^{T}(\mathbf{i}[m]-\mathbf{h} \widehat{\boldsymbol{\eta}})=\boldsymbol{\ell}_{\mathbf{I}}^{\dagger}(\mathbf{i}[m]-\mathbf{h} \widehat{\boldsymbol{\eta}})
$$

for $m=1, \ldots, M$, with $\ell$ given in (20). The static polynomial coefficients of $s_{k}$ in (5) are deduced as

$$
\overline{\boldsymbol{\theta}}=\frac{1}{M} \sum_{m=1}^{M} \widehat{\boldsymbol{\theta}}_{m} .
$$

Alternatively, one can show that

$$
\overline{\boldsymbol{\theta}}=\ell_{\mathbf{I}}^{\dagger}(\overline{\mathbf{i}}-\mathbf{h} \widehat{\boldsymbol{\eta}}) .
$$

With a set of measured INL sequences corresponding to the frequencies $f_{1}, \ldots, f_{M}$ (in hertz), the solution for the static HCF centered segments, dynamic LCF polynomial, and static LCF polynomial are given by (35), (37), and (39), respectively. For a frequency $f_{m}$, the estimated INL sequence is now given by

$$
\widehat{\mathbf{i}}_{m}=\mathbf{h} \widehat{\boldsymbol{\eta}}+\boldsymbol{\ell} \widehat{\boldsymbol{\theta}}_{m}=\underbrace{\mathbf{h} \widehat{\boldsymbol{\eta}}+\boldsymbol{\ell} \overline{\boldsymbol{\theta}}}_{\text {static part }}+\underbrace{\ell \tilde{\boldsymbol{\theta}}_{m}}_{\text {dynamic part }}
$$

where $\tilde{\boldsymbol{\theta}}_{m}$ is the parameter vector that represents the polynomial model of the LCF at a single frequency $f_{m}$, i.e.,

$$
\tilde{\boldsymbol{\theta}}_{m}=\widehat{\boldsymbol{\theta}}_{m}-\overline{\boldsymbol{\theta}}
$$


The methodology used to determine the number and sizes of the piecewise-linear segments and the LCF order $L$ is presented in the next section.

\section{HCF SEgmentation AND LCF MOdEL ORDER DETERMINATION}

The polynomial order $L$ of the LCF model, the set of $P$ segments in the HCF model, and their borders can methodically be determined. Previous work on the topic used an ad hoc method to determine the number of segments and their respective borders [17], [18]. Furthermore, substantial manual intervention was used to acquire the final set of segments.

\section{A. Cost Function for Segmentation}

The HCF is a static quantity that is modeled by a set of centered segments. The number of segments $P$ and their borders $k_{1}, \ldots, k_{P-1}$ are to be determined. A cost function $F(\mathbf{h})$ to minimize is the norm of the error vector between the average INL data in (36) and the static model $\mathbf{h} \widehat{\boldsymbol{\eta}}+\boldsymbol{\ell} \overline{\boldsymbol{\theta}}$ in (40), i.e.,

$$
F(\mathbf{h})=\|\overline{\mathbf{i}}-\ell \overline{\boldsymbol{\theta}}(\mathbf{h})-\mathbf{h} \widehat{\boldsymbol{\eta}}(\mathbf{h})\| .
$$

From the calculated mean INL data $\overline{\mathbf{i}}$, two terms are subtracted: the static part of the LCF model and the HCF component. It is worth noting that the quantities $\overline{\boldsymbol{\theta}}(\mathbf{h})$ and $\widehat{\boldsymbol{\eta}}(\mathbf{h})$ are the same as those given in (35) and (38), respectively, but the $\mathbf{h}$ dependence is made explicit here because it is the optimization variable. The term $\mathbf{h}$ is the block diagonal matrix (11), where the subvectors $\mathbf{h}_{p}$ in (12) are formulated in terms of the $p$ th segment, which should all be optimized in width and number.

Inserting $\overline{\boldsymbol{\theta}}$ given by (39) into (42) yields

$$
F(\mathbf{h})=\left\|\overline{\mathbf{i}}-\ell \ell_{\mathbf{I}}^{\dagger} \overline{\mathbf{i}}+\ell \ell_{\mathbf{I}}^{\dagger} \mathbf{h} \widehat{\boldsymbol{\eta}}(\mathbf{h})-\mathbf{h} \widehat{\boldsymbol{\eta}}(\mathbf{h})\right\| .
$$

Using $\boldsymbol{\pi}_{\perp}$ given in (32), (43) can be rewritten as

$$
F(\mathbf{h})=\left\|\boldsymbol{\pi}_{\perp}(\overline{\mathbf{i}}-\mathbf{h} \widehat{\boldsymbol{\eta}}(\mathbf{h}))\right\| .
$$

Because the HCF model data are a structure of segments, the employed polynomial order $L$ (rank of $\ell$ ) is far too small to characterize the HCF model data (except for a dc level); therefore, $\boldsymbol{\pi}_{\perp} \mathbf{h} \widehat{\boldsymbol{\eta}}(\mathbf{h}) \approx \mathbf{h} \widehat{\boldsymbol{\eta}}(\mathbf{h})$. On the other hand, the average INL data $\overline{\mathbf{i}}$ contain a significant polynomial LCF component. By introducing $\tilde{\mathbf{i}}$

$$
\tilde{\mathbf{i}}=\boldsymbol{\pi}_{\perp} \overline{\mathbf{i}}=\overline{\mathbf{i}}-\ell \ell_{I}^{\dagger} \overline{\mathbf{i}}
$$

The projected set of data (45) resembles an LCF-free INL, which is HCF. It is worth mentioning that $\tilde{\mathbf{i}}$ is a zero-centered data sequence. Using (45) and (35) into (44) results in

$$
F(\mathbf{H})=\left\|\tilde{\mathbf{i}}-\mathbf{h}\left(\mathbf{h}^{T} \boldsymbol{\pi}_{\perp} \mathbf{h}\right)^{-1} \mathbf{h}^{T} \boldsymbol{\pi}_{\perp} \overline{\mathbf{i}}\right\|
$$

where $\mathbf{h}\left(\mathbf{h}^{T} \boldsymbol{\pi}_{\perp} \mathbf{h}\right)^{-1} \mathbf{h}^{T} \boldsymbol{\pi}_{\perp} \overline{\mathbf{i}}$ is the data resulting from a weighted least-squares solution; the task of $\boldsymbol{\pi}_{\perp}$ is to phase out the effect of the polynomial attribute in the mean INL prior to the HCF segments fit. Thus, for simplicity, the $\pi_{\perp}$ entity in (46) can be replaced by the identity matrix and the mean INL data

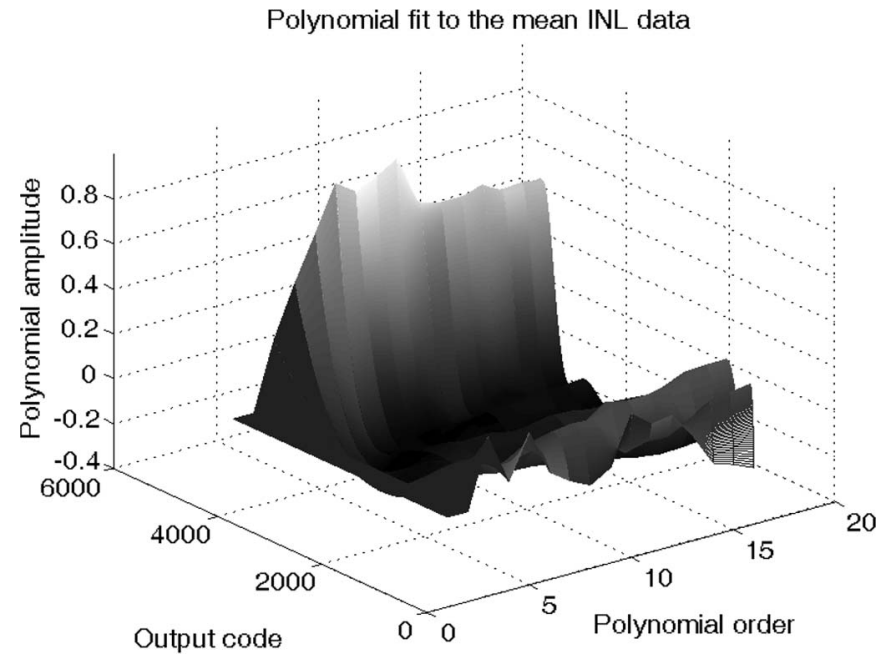

Fig. 5. Polynomial fit to average INL data versus polynomial order $L$. The shape of the polynomial tends to settle after the fifth order.

$\overline{\mathbf{i}}$ by the centered $\mathrm{HCF}$ data $\tilde{\mathbf{i}}$. As a result, the estimated $\mathrm{HCF}$ parameters can be approximated by the least-squares solution of the centered INL data $\tilde{\mathbf{i}}$, i.e.,

$$
\widehat{\boldsymbol{\eta}}(\mathbf{h}) \approx\left(\mathbf{h}^{T} \mathbf{h}\right)^{-1} \mathbf{h}^{T} \tilde{\mathbf{i}}=\mathbf{h}_{\mathbf{I}}^{\dagger} \tilde{\mathbf{i}} .
$$

A simplified cost function $F^{\prime}(\mathbf{h})$ to minimize is

$$
F^{\prime}(\mathbf{h})=\left\|\tilde{\mathbf{i}}-\mathbf{h}\left(\mathbf{h}^{\mathbf{T}} \mathbf{h}\right)^{-1} \mathbf{h}^{\mathbf{T}} \tilde{\mathbf{i}}\right\| .
$$

The cost function (48) is used in the following to tune the segment borders $k_{1}, \ldots, k_{P-1}$.

\section{B. LCF Polynomial Order L}

The segmentation criterion is formulated in terms of the $\mathrm{HCF}$ component $\mathbf{h}$ and the zero-centered HCF data $\tilde{\mathbf{i}}$ produced by the projection $\tilde{\mathbf{i}}=\pi_{\perp} \overline{\mathbf{i}}$, which cancels any polynomial shape of the averaged INL data up to a specified polynomial order $L$. The LCF polynomial order $L$ must be large enough to be able to capture the polynomial attributes of the INL data, which generates a residual sequence (45) that mimics the HCF structure. However, if the LCF order $L$ is further increased, the LCF polynomial tends to represent the HCF structure as well. Accordingly, the order $L$ must be selected in a tradeoff, so that the estimated polynomial depicts the smooth behavior of the INL data but keeps HCF modeling to the segmented model. If the LCF data can be assumed to be perfectly described by a polynomial of order $L_{0}$, then applying the projection matrix $\boldsymbol{\pi}_{\perp}$ to the data will result in the zero vector if $L$ that determines $\boldsymbol{\pi}_{\perp}$ fulfills $L \geq L_{0}$. Fig. 5 shows the resulting polynomial fitted to the sample INL data versus polynomial order $L$. Obviously, by increasing the model order $L$, the polynomial is able to better mimic INL data, but after the fifth order, the polynomial is more or less unaffected by the choice of $L$. A measure of the fit is the RMS error

$$
\sigma_{L}=\sqrt{\frac{\|\tilde{\mathbf{i}}\|^{2}}{2^{N}-1}} .
$$




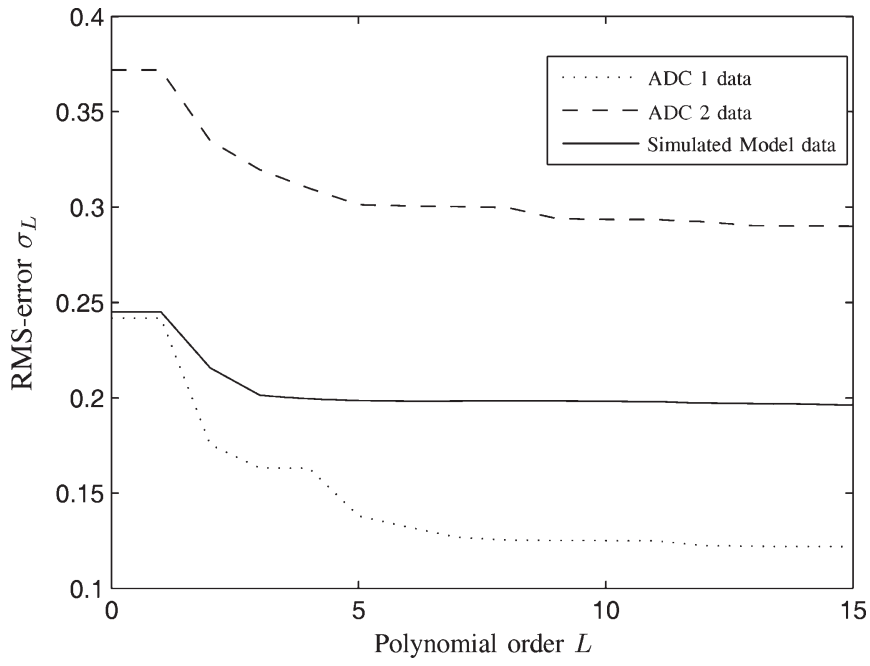

Fig. 6. RMS error of polynomial fit versus LCF polynomial order $L$. Because the INL data are adjusted for gain and offset, there is no difference in the results for $L=0$ and $L=1$. The difference in absolute values is because of the different noise levels in the experimental and simulated data.

The RMS error $\sigma_{L}$ is plotted in Fig. 6 as a function of the order $L$. Simulated data originating from the simulation model provided by the ADC manufacturer, as well as experimental data from two ADC evaluation boards of the same model, are used. It is obvious from the diagram in Fig. 6 that the magnitude of the residual HCF sequence begins to stabilize after the fifth order for the measured data. Thus, we can deduce from Figs. 5 and 6 that $L \geq 5$-order polynomial is needed to trade between LCF and HCF modeling. A low complexity model implies a polynomial of the lowest order (i.e., $L=5$ ), but to maintain a safety margin, $L$ is chosen to be $L=7$ throughout this paper. Polynomial order selection criteria, namely, trading polynomial fit and order, which are variants of the Akaike information criteria [20], are possible; however, they are beyond the scope of this paper. Analysis of such criteria is not straightforward, because of the actual undermodeling when determining the polynomial order $L$.

\section{Determining the Number of HCF Segments $P$}

To determine the number of HCF segments $P$, recall the piecewise-linear shape of the HCF, as illustrated in Fig. 4. Furthermore, recall that the $\mathrm{HCF}$ is a static behavior of the ADC. Thus, the segmentation relies on the average INL data $\overline{\mathbf{i}}$ from which the polynomial LCF variations have been removed by the $\pi_{\perp}$ projection, which is the resulting $\tilde{\mathbf{i}}$ for a suitable model order $L$. The resulting data describing the $\mathrm{HCF}$ are still quite noisy, which is evident from, e.g., Fig. 3. To denoise the data and find the segment borders, several approaches are possible. One alternative is to use local projection with a firstorder projection matrix and look for the transients that occur at the segment borders. Another similar approach is to use a filter to smooth the data. A low-pass filter is applied to the HCF data of (45). Best practice indicates that convolving $\tilde{\mathbf{i}}$ with a triangular window that is approximately half of the average segment length is appropriate. Here, the triangular window is of dimension 127. A longer window masks multiple segments,

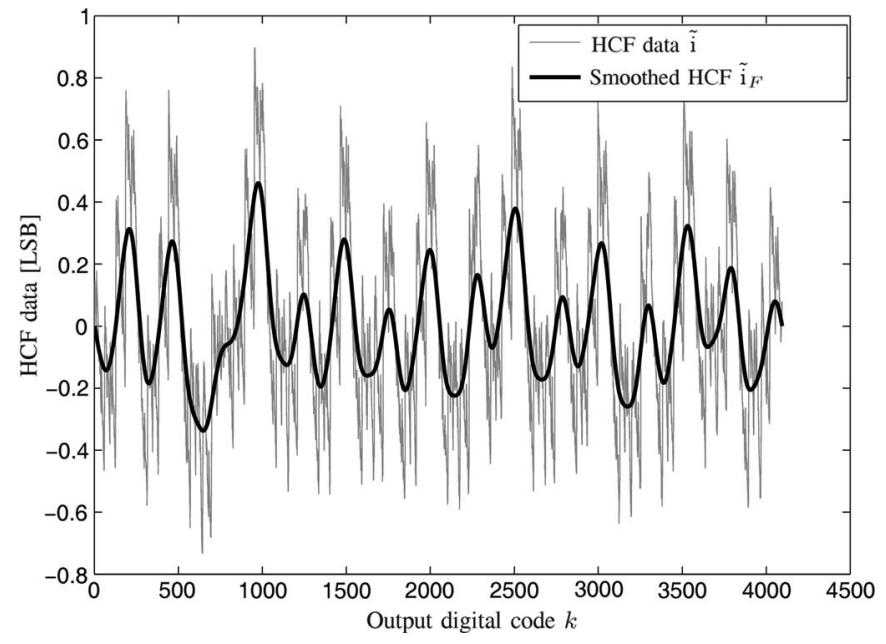

Fig. 7. Filtered version $\tilde{\mathbf{i}}_{F}$ of calculated HCF data $\tilde{\mathbf{i}}$, obtained from the measured average INL $\overline{\mathbf{i}}$, as a function of the output digital code $k$.

TABLE I

Algorithm for Selecting the HCF Number of Segments $P$ And Initial Guess About Their Borders $\left\{k_{p}\right\}$. Negative Slopes

1) Set the first segment border at $k_{0}=1$.

2) Select the code bin $k^{\prime}$ representing the $\tilde{\mathbf{i}}_{F}$ minimum between the first bin $k=1$ and first zero crossing which yields $k_{1}=k^{\prime}+1$.

3) Select the code bin representing the $\tilde{\mathbf{i}}_{F}$ minimal value between the second and third zero crossing as the border of the third segment.

4) Continue by choosing the segments borders as the minimum $\tilde{\mathbf{i}}_{F}$ value between each two consecutive disjoint zero crossings until the last code bin $k=2^{N}-1$ is reached.

5) The number of segments $P$ is obtained by adding 1 to the number of zero crossings.

and a shorter window traces the small-scale structures and noise. An example of the resulting smoothed HCF data that forms the basis for the segmentation is shown in Fig. 7. By filtering the data in the forward and reverse directions, the phase information is preserved.

The filtered sequence $\tilde{\mathbf{i}}_{F}$ is used to determine the number of segments $P$ and a reasonable initial guess about their borders, which are $k_{p}$ values. The algorithm to calculate $P$ from $\tilde{\mathbf{i}}_{F}$ is summarized in Table I, where it is assumed that a negative slope is common for all the centered segments in the case of the measured data. (A similar algorithm can be derived assuming positive slopes.) The obtained $P$ and the corresponding associated segments borders $k_{1}, \ldots, k_{P-1}$ are then fed into a numerical optimizer to perform fine-tuning by minimizing (48).

\section{HCF Segment Fine-Tuning}

Having roughly segmented the data, the remaining task is to optimize their respective widths, that is, to fine-tune the $k_{p}$ values. Thus, for a given number $P$ of segments, we consider

$$
\left(\widehat{k}_{1}, \ldots, \widehat{k}_{P-1}\right)=\arg \min F^{\prime}(\mathbf{h})
$$

where $F^{\prime}(\mathbf{h})$ was defined in (48). The optimization process can be done using numerical software such as MATLAB, with the initial $k_{p}$ values obtained as indicated in the previous section. 
TABLE II

INL CHARACTERIZATION

1) Collect the vector INL data $\mathbf{i}[1], \ldots, \mathbf{i}[M]$ corresponding to test frequencies $f_{1}, \ldots, f_{M}[\mathrm{~Hz}]$ and compute the average INL $\overline{\mathbf{i}}$ as in (36).

2) Determine LCF model order $L$ from $\sigma_{L}$ in (49) based on the projected INL-average $\tilde{\mathbf{i}}$ as in (45).

3) Determine the number of segments $P$ and initial guesses of the segment borders from the filtered data $\tilde{\mathbf{i}}_{F}$ by using the algorithm given in Tab. I.

4) Construct the matrix $\mathbf{h}$ in (11) as the initial guess and perform the optimization in (50) to obtain the optimized segment set.

5) Use the new set of segments to reconstruct the $\mathbf{h}$ matrix.

6) Calculate the HCF $\widehat{\boldsymbol{\eta}}$ and LCF static $\overline{\boldsymbol{\theta}}$ and dynamic $\widehat{\boldsymbol{\theta}}_{m}$ parameters according to (35)-(38).

7) Calculate the static model as $\mathbf{h} \widehat{\boldsymbol{\eta}}+\boldsymbol{\ell} \overline{\boldsymbol{\theta}}$, where $\boldsymbol{\ell}$ is defined in (20).

8) For $m=1$ to $M$ :

Calculate the dynamic LCF as $\boldsymbol{\ell}\left(\widehat{\boldsymbol{\theta}}_{m}-\overline{\boldsymbol{\theta}}\right)$.

The MATLAB function "fmincon," which finds a constrained minimum of a function of several variables, is used. The constraints, variables, and initial guesses are used as input into the "optimset" function. A minimum step of one code bin is allowed to the function, and the optimized solution (or, equivalently, the segment borders) is rounded after each iteration. Thus, a different integer number is obtained at each iteration.

Once the proper $L, P$, and optimized set of $k_{p}$ 's are found, the LCF and HCF components of the INL data are obtained by solving the least-squares problem studied in Section III. The methodology for segmentation and least-squares estimation is summarized in Table II.

\section{EXPERIMENTAL INVESTIGATION}

\section{A. ADC Under Test}

The ADC under test is a commercial 12-bit pipeline Analog Devices AD9430 intended for direct intermediate frequency sampling, which operates up to a 210-MSPS conversion rate and has a spurious-free dynamic range (SFDR) ranging from 70 to $88 \mathrm{dBc}$ at $0.5 \mathrm{dBFS}$ for frequencies within the bandwidth, according to the data sheet. Two different samples of the ADC under test were employed (referred to as ADC1 and ADC2, respectively), as well as simulated AD9430 data generated by the simulation tools provided by the manufacturer. The ADC performs best in terms of SFDR between 65 and $75 \mathrm{MHz}$. A typical SFDR value is $80 \mathrm{dBc}$ at $70 \mathrm{MHz}$. The signal-to-noise ratio is typically $65 \mathrm{~dB}$, and the effective number of bits is 10.6 bits at $70 \mathrm{MHz}$.

\section{B. Experimental Setup}

The clock and input signals are generated by a high-quality Rohde \& Schwartz SMU200A vector signal generator (VSG). More information about the test bed can be found in [21]. A noncommercial wideband (30-300 MHz) low-distortion amplifier with a gain of $14.3 \mathrm{~dB}$ is used at the output of the latter to avoid overdriving the VSG. Mini-Circuits SLP filters are used at the input of the ADC under test to attenuate any potential harmonics or spurious signals. It was verified that all
TABLE III

ADC MEASUREMENT SETUP

\begin{tabular}{cccc}
\hline$m$ & Frequency [Hz] & SLP- Filter & Passband [MHz] \\
\hline 1 & 30004597 & $30+$ & 32 \\
\hline 2 & 35003227 & $30+$ & 32 \\
\hline 3 & 40001857 & $50+$ & 48 \\
\hline 4 & 45000487 & $50+$ & 48 \\
\hline 5 & 50076019 & $50+$ & 48 \\
\hline 6 & 55074649 & $50+$ & 48 \\
\hline 7 & 60124547 & $70+$ & 60 \\
\hline 8 & 65071909 & $70+$ & 60 \\
\hline 9 & 70121807 & $70+$ & 60 \\
\hline 10 & 75069169 & $100+$ & 98 \\
\hline 11 & 80067799 & $100+$ & 98 \\
\hline 12 & 85066429 & $100+$ & 98 \\
\hline 13 & 90065059 & $100+$ & 98
\end{tabular}

the measured harmonics lie in the stopband (at least $40-\mathrm{dB}$ attenuation) of the filters. Table III summarizes the set of measured frequencies, the filters in use, and their passbands. The measured frequencies are guaranteed to be a noninteger submultiple of the sampling frequency. For the $75-$ and $80-\mathrm{MHz}$ frequencies, the SLP100+ filter in use was unable to sufficiently attenuate (up to $-80 \mathrm{dBFS}$ ) the harmonics generated, because they occurred in a stopband of $20-\mathrm{dB}$ attenuation. A surface acoustic wave passband (50-90 MHz) TRIQUINT (SAWTEK854680) filter encapsulated and matched by Billius Mätteknik was also used to further attenuate the harmonics. Because the latter filter has an insertion loss of $21.7 \mathrm{~dB}$, an additional amplifier (identical to the first amplifier) was used to compensate for the power loss. The amplifiers and filters are specially matched by Billius Mätteknik to a $50-\Omega$ load, as for the VSG in use. All the measured signals are verified (by the signal analyzer) to be at least $-80 \mathrm{dBFS}$.

The power of each test signal is tuned to a signal overload of 5 LSBs, which is the recommended input power to calculate the INL [22]. Three ADC output sequences of length $2^{18}$ samples (i.e., $>2^{N} \cdot \pi$ [7]) are measured for each frequency. The measured data are saved, and their INL is calculated by the histogram test [7] (see also [2] and [6]). Basically, the INL for each frequency is the average of three INL sequences, where each is the outcome of the histogram test at the given frequency. Averaging reduces the effect of noise on the obtained INL.

\section{Static INL}

The developed methodology was applied on both simulated and measured data. All parametric models comprised $P=16$ segments and made use of an $L=7$ th-order polynomial, thus making the number of parameters for the static model equal to 24. The complete static part of the resulting INL models is presented in Fig. 8, with the original INL data shown as a reference.

Similar behavior is common for the models that represent the simulated and measured data from the two ADCs. The three static polynomials have similar magnitudes, although the models that represent the measured data show a more pronounced decay at high-code levels, particularly for ADC1. Compared with the model representing the simulated data, the model representing $\mathrm{ADC} 2$ is more structured than the $\mathrm{ADC} 1$ 

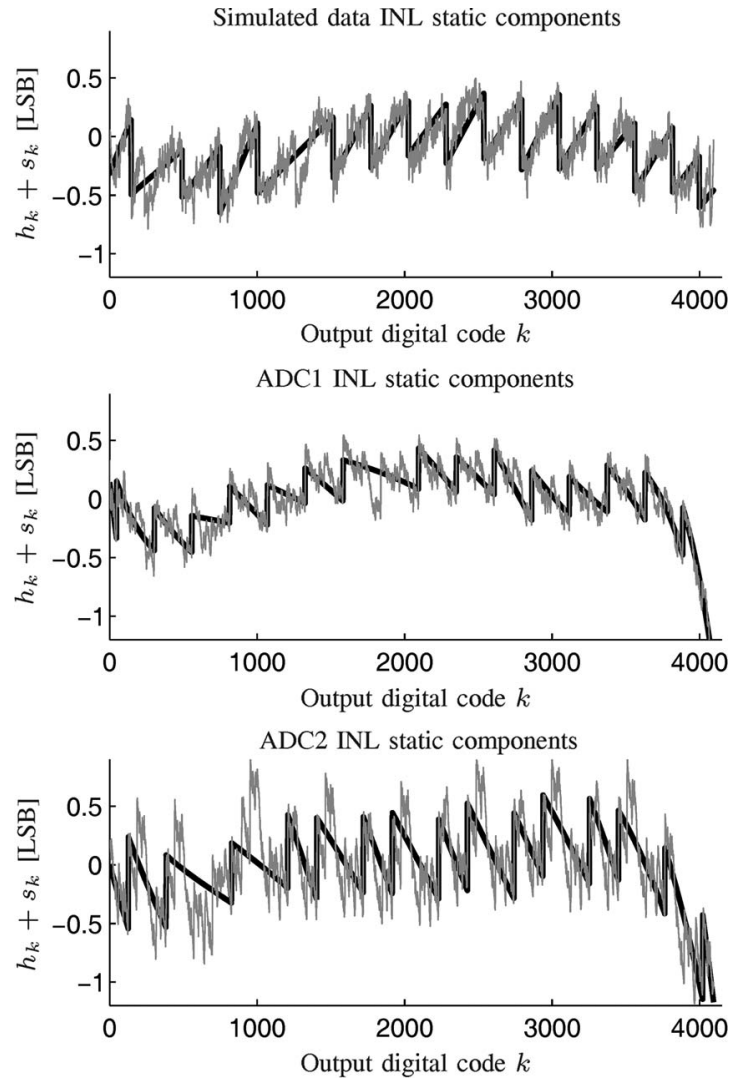

Fig. 8. Static components of the ADC INL in LSBs as a function of the output digital code $k$. In each diagram, the resulting parametric model is shown with a black line, whereas the averaged measured INL is plotted with a gray line.

model. The HCF segments in the ADC2 model are more similar in size and structure to the simulated model when compared with those from ADC1. However, ADC1 HCF data are smaller in magnitude than those from $\mathrm{ADC} 2$; thus, the former are able to capture more of the information in the measured INL in comparison with the latter (see also Fig. 10). One notable difference between the resulting data models is the common slope of the HCF segments, which is positive for the data provided by the manufacturer simulation tool but negative for the measured data. We currently have no explanation for this difference.

\section{Dynamic Part of the $L C F$}

The measured data were composed of $M=13$ stimuli frequencies, where the dynamic part of the LCF was modeled by an $L=7$ th-order polynomial. Thus, the dynamic model comprises 104 (that is $13 \times 8$ ) parameters.

The dynamic part of the LCF component is plotted in Fig. 9. From the displayed results, one may note that the simulation model provided by the manufacturer indeed captures the input frequency dependence of the AD9430 performance. However, this dependence is more obvious in the case of the measured ADC data INL. These results are in accordance with the data sheet, where the performance varies with the frequency. One may also note that the dynamic effects on the INL are not as pronounced as the static effects, as further investigated in the following discussion.
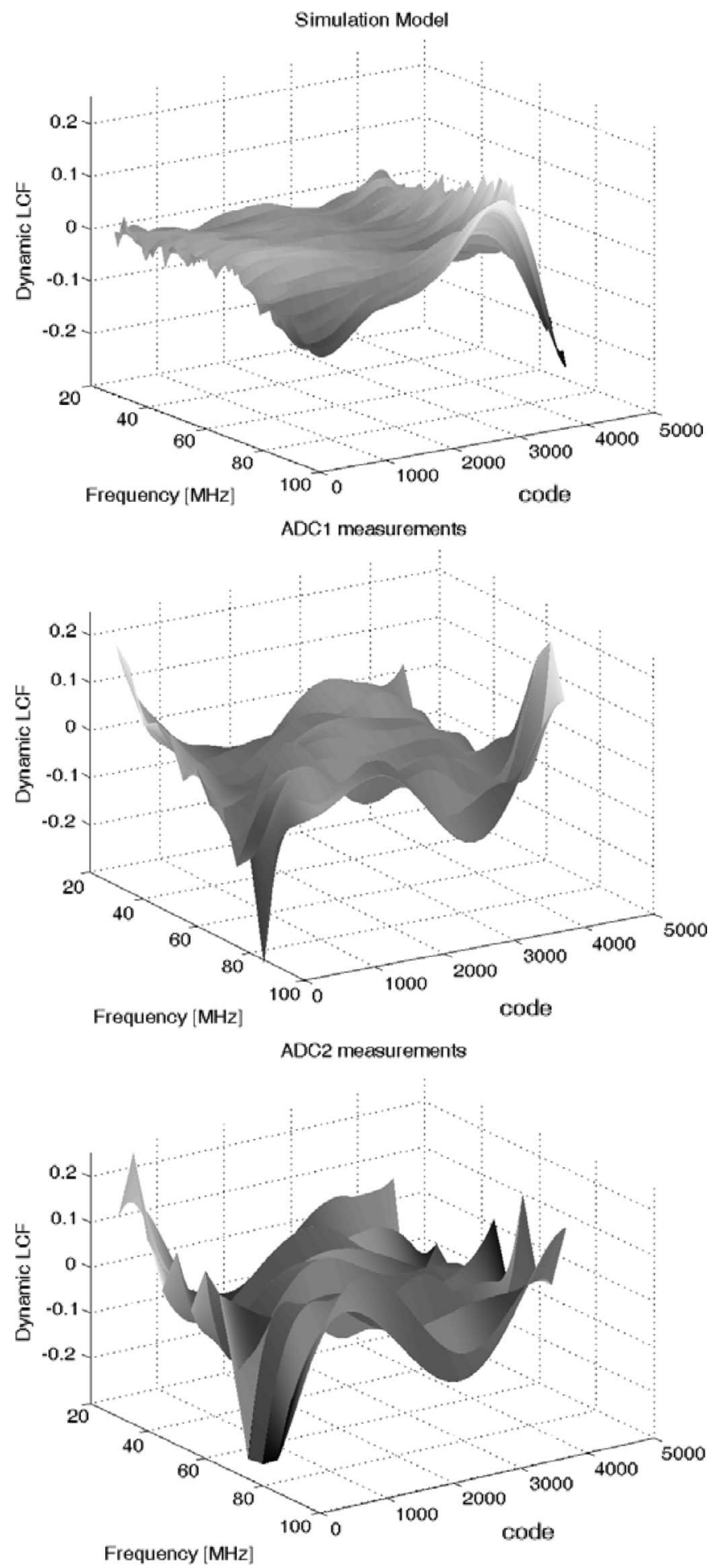

Fig. 9. Dynamic component of the LCF measured in LSBs as a function of the sine-wave test frequency $f_{m}$ (in megahertz) and the output digital code $k$.

\section{E. Influence of the Model Structure on INL Modeling}

The extent to which the different captured INL terms describe the measured INL is an interesting question. For a given test frequency $f_{m}$, the RMS error per test frequency is given by

$$
\sigma_{\mathrm{SLCF}}[m]=\sqrt{\frac{1}{2^{N}-1} \sum_{k=1}^{2^{N}-1}\left(i[m, k]-\widehat{s}_{k}\right)^{2}}
$$



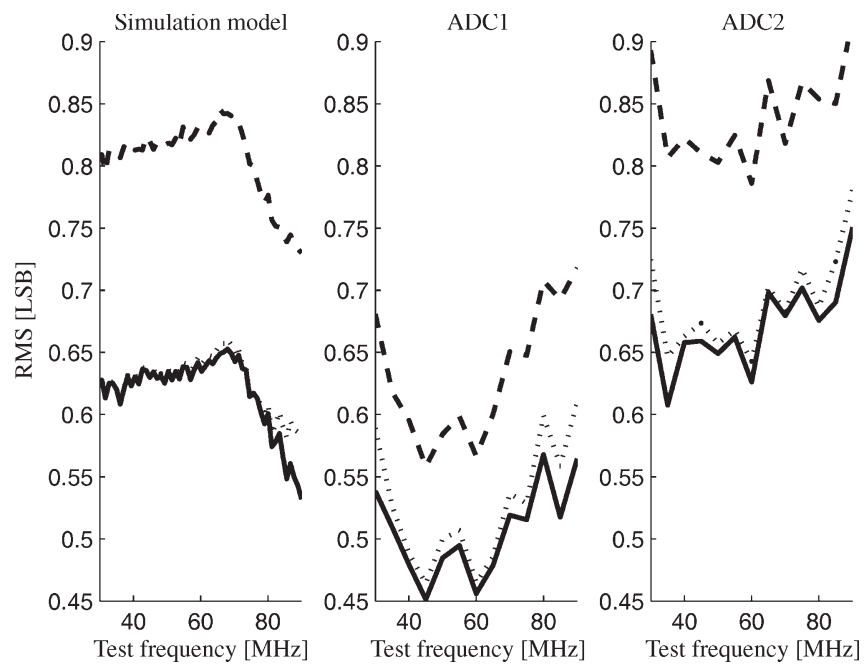

Fig. 10. Capacity of the different models to capture the behavior of the measured INL. From top to bottom: $\sigma_{\mathrm{SLCF}}[\mathrm{m}] / \sigma_{\mathrm{INL}}[\mathrm{m}]$ (dashed line), $\sigma_{\text {Static model }}[m] / \sigma_{\text {INL }}[m]$ (dotted line), and $\sigma_{\text {Full model }}[m] / \sigma_{\text {INL }}[m]$ (solid line).

where $i[m, k]$ is the measured INL, and $\widehat{s}_{k}$ denotes the estimated static part of the LCF, which, in other words, is the amount of the INL behavior that is captured by the (here, $L=7$ th-order) polynomial model. As a first refinement, adding the $P$-parameter HCF model (here, $P=16$ ), we study

$$
\sigma_{\text {Static model }}[m]=\sqrt{\frac{1}{2^{N}-1} \sum_{k=1}^{2^{N}-1}\left(i[m, k]-\widehat{s}_{k}-\widehat{h}_{k}\right)^{2}} .
$$

Finally, adding the estimated $L \times M$-parameter dynamic LCF model, the figure of merit is

$$
\sigma_{\text {Full model }}[m]=\sqrt{\frac{1}{2^{N}-1} \sum_{k=1}^{2^{N}-1}\left(i[m, k]-\widehat{s}_{k}-\widehat{h}_{k}-\widehat{d}_{m, k}\right)^{2}} .
$$

The resulting figures of merit are displayed in Fig. 10 for the considered ADC data. As a reference, the RMS value of the measured INL is also considered, i.e.,

$$
\sigma_{\mathrm{INL}}[m]=\sqrt{\frac{1}{2^{N}-1} \sum_{k=1}^{2^{N}-1} i[m, k]^{2}} .
$$

From the results displayed in Fig. 10, one may note three observations.

1) For the simulated data, we can deduce that the static components $\left(\widehat{s}_{k}\right.$ and $\left.\widehat{h}_{k}\right)$ equally reduce the RMS error because the (static) polynomial is of the same order of magnitude as the segments (cf. Fig. 8). The role of the dynamic component is not relevant until the higher test frequencies, at which the dynamic polynomial is more pronounced (Fig. 9).

2) The preceding observation also holds true for the measured data; the static components capture most of the INL behavior, but the proportions between the two static terms are not equal in this case. For the first ADC, the polynomial LCF $\widehat{s}_{k}$ has the most significant role in reducing the RMS error.

3) The effects of the dynamic term are more pronounced for the real ADCs compared with the simulation model. However, the effect is most significant at high frequencies, but an increased effect of the dynamic model is also noted at low frequencies.

\section{CONCLUSION}

The INL of an ADC is a significant figure of merit. In this paper, a methodology to model the input frequency-dependent characteristics of the INL has been proposed and thoroughly investigated. Based on the inherent structure of nowadays pipelined ADCs for RF applications, a parametric model is proposed that is linear in its parameters. The least-squares problem is studied in detail, including the model order selection criteria. In short, the INL is decomposed into three terms: two that describe the input-independent or static behavior of the $\mathrm{ADC}$ and one that describes the input frequency-dependent part. The methodology is applied to a nowadays 12-bit ADC running at 210 MSPS. Using only eight parameters, a large portion of the INL behavior is captured by the model, and with 24 parameters, the majority of the behavior is captured. By adding an input-dependent term with an additional 96 parameters, further improvement is gained. Depending on the application, suitable parts of the model are considered. For data-sheet presentation, the full model is a versatile tool to illustrate the frequency-dependent characteristics of the ADC. The obtained INL models are also believed to be powerful for INL-based postcorrection of ADCs for broadband applications; this latter topic will be considered for future studies.

\section{REFERENCES}

[1] K. M. Mukund, S. Seshadri, J. Devarajulu, and M. Kannan, "A $1 \mathrm{GHz}$ pipelined low power floating point arithmetic unit with modified scheduling for high speed applications," IEEE Trans. Instrum. Meas., vol. 56, no. 3, pp. 377-381, Jun. 2007.

[2] S. Rapuno, P. Daponte, E. Balestrieri, L. de Vito, S. J. Tilden, S. Max, and J. Blair, "ADC parameters and characteristics-Part 6 in a series of tutorials in instrumentation and measurement," IEEE Instrum. Meas. Mag., vol. 8, no. 5, pp. 44-54, Dec. 2005.

[3] W. Kester, Analog-Digital Conversion. Norwood, MA: Analog Devices, 2004.

[4] T. Kuyel and H. Bilhan, "Relating linearity test results to design flaws of pipelined analog-to-digital converters," in Proc. Int. Test Conf., Atlantic City, NJ, Sep. 1999, pp. 772-779.

[5] B. Provost and E. Sanchez-Sinencio, "A practical self-calibration scheme implementation for pipeline ADC," IEEE Trans. Instrum. Meas., vol. 53 , no. 2, pp. 448-456, Apr. 2004.

[6] T. E. Linnenbrink, J. Blair, S. Rapuno, P. Daponte, E. Balestrieri, L. de Vito, S. Max, and S. J. Tilden, "ADC testing-Part 7 in a series of tutorials in instrumentation and measurement," IEEE Instrum. Meas. Mag., vol. 9, no. 2, pp. 37-47, Apr. 2006.

[7] IEEE Standard for Terminology and Test Methods for Analog-to-Digital Converters, IEEE Std. 1241-2000, 2001.

[8] L. Michaeli, P. Michalko, and J. Saliga, "Identification of unified ADC error model by triangular testing signal," in Proc. 10th Workshop ADC Modelling Testing, Gdynia/Jurata, Poland, 2005, pp. 605-610.

[9] L. Michaeli, P. Michalko, and J. Saliga, "ADC testing by decomposition of the error model," Measurement, vol. 5, pp. 39-42, 2005, sec. 1.

[10] A. Cruz Serra, F. Alegria, L. Michaeli, P. Michalko, and J. Saliga, "Fast ADC testing by repetitive histogram analysis," in Proc. IEEE Instrum. Meas. Technol. Conf., Apr. 24-27, 2006, pp. 1633-1638. 
[11] N. Björsell and P. Händel, "Achievable ADC performance by postcorrection utilizing dynamic modeling of the integral nonlinearity," EURASIP J. Adv. Signal Process., vol. 2008, no. 7, p. 10, 2008, Article ID 497187, DOI: $10.1155 / 2008 / 497187$

[12] L. Michaeli, P. Michalko, and J. Saliga, "Identification of ADC error model by testing of the chosen code bins," in Proc. 12th IMEKO TC4 Int. Symp., Zagreb, Croatia, Sep. 25-27, 2002, pp. 132-137.

[13] P. Arpaia, P. Daponte, and L. Michaeli, "A dynamic error model for integrating analog-to-digital converters," Measurement, vol. 25, no. 4, pp. 255-264, Jun. 1999.

[14] L. Michaeli, P. Michalko, and J. Saliga, "Unified ADC nonlinearity error model for SAR ADC,” Measurement, vol. 41, no. 2, pp. 198-204, Feb. 2008.

[15] A. C. Serra, M. F. da Silva, P. M. Ramos, R. C. Martins, L. Michaeli, and J. Saliga, "Combined spectral and histogram analysis for fast ADC testing," IEEE Trans. Instrum. Meas., vol. 54, no. 4, pp. 1617-1623, Aug. 2005.

[16] N. Björsell and P. Händel, "Dynamic behavior models of analog to digital converters aimed for post-correction in wideband applications," in Proc. 11th Workshop ADC Modelling Testing, Rio de Janerio, Brazil, Sep. 17-22, 2006.

[17] P. Händel, N. Björsell, and M. Jansson, "Model based dynamic characterization of analog-digital-converters at radio frequency-Invited paper," in Proc. Int. Conf. Signal Process. Appl., Sharjah, UAE, Feb. 12-15, 2007, pp. 1-6.

[18] S. Medawar, N. Björsell, P. Händel, and M. Jansson, "Dynamic characterization of analog-digital-converters non-linearities," in Proc. Mosharaka Int. Conf. Wireless Commun. Mobile Comput., Amman, Jordan, Sep. 6-8, 2007.

[19] S. Medawar, P. Handel, N. Bjorsell, and M. Jansson, "ADC characterization by dynamic integral non-linearity," in Proc. 13th Workshop ADC Modeling Testing, Florence, Italy, Sep. 22-24, 2008, pp. 1-6.

[20] H. Bozdogan, "Model selection and Akaike's Information Criterion (AIC): The general theory and its analytical extensions," Psychometrika, vol. 52, no. 3, pp. 345-370, Sep. 1987.

[21] N. Björsell, O. Anderson, and P. Händel, "High dynamic test-bed for characterization of analogue-to-digital converters up to 500 MSPS," in Proc. 10th Workshop ADC Modeling Testing, Gdynia, Poland, 2005, pp. 601-604.

[22] J. Blair, "Histogram measurement of ADC nonlinearities using sine waves," IEEE Trans. Instrum. Meas., vol. 43, no. 3, pp. 373-383, Jun. 1994.

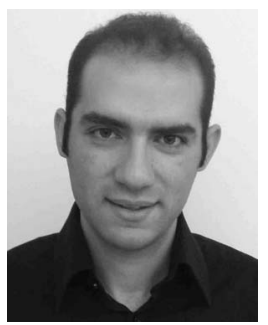

Samer Medawar received the M.E. degree in electrical engineering from the Lebanese University ULFG2, Beirut, Lebanon, in 2005 and the M.Sc. degree in electrical engineering and telecommunications from the University of Gävle, Gävle, Sweden, in 2006. He is currently working toward the Ph.D. degree with the Signal Processing Laboratory, ACCESS Linnaeus Center, Royal Institute of Technology, Stockholm, Sweden. He did his M.Sc. thesis project at the Mobile Communications Group, Aalborg University, Aalborg, Denmark.

His main research interests are ADC error characterization, modeling, and postcorrection, in addition to RF and antenna measurements and processing.

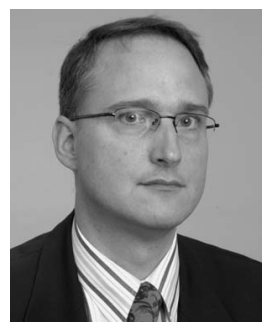

Peter Händel (S'88-M'94-SM'98) received the Ph.D. degree from Uppsala University, Uppsala, Sweden, in 1993.

From 1987 to 1993, he was with Uppsala University. From 1993 to 1997, he was with Ericsson AB, Kista, Sweden. From 1996 to 1997, he was also with Tampere University of Technology, Tampere, Finland. Since 1997, he has been with the Royal Institute of Technology, Stockholm, Sweden, where he is currently a Professor of signal processing with the Signal Processing Laboratory, ACCESS Linnaeus Center. From 2000 to 2006, he was with the Swedish Defense Research Agency. He is currently a Guest Professor with the University of Gävle, Gävle, Sweden. He has served as a member of the editorial board of the EURASIP Journal of Advances in Signal Processing. He has also served as a member of the editorial advisory board of Recent Patents on Electrical Engineering. He is a member of the editorial board of Hindawi's Research Letters in Signal Processing and Journal of Electrical and Computer Engineering.

Dr. Händel has served as an Associate Editor of the IEEE TRANSACTIONS ON Signal PROCESSING.

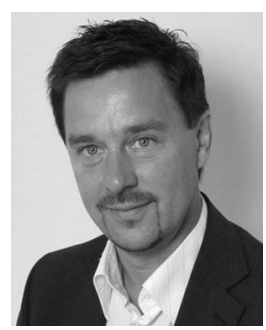

Niclas Björsell (S'02-M'08) was born in Falun, Sweden, in 1964. He received the B.Sc. degree in electrical engineering and the Licentiate degree in automatic control from Uppsala University, Uppsala, Sweden, in 1994 and 1998, respectively, and the Ph.D. degree in telecommunication from the Royal Institute of Technology, Stockholm, Sweden, in 2007.

He has several years of experience from research and development projects that fostered collaborations between industry and the academy. He has worked as a Project Manager for some of the R\&D projects. Since 2006, he has been the Head of the Division of Electronics, Department of Technology and Built Environment, University of Gävle, Gävle, Sweden. He has published more than 20 papers in journals and conferences. His research interests include radio frequency measurement technology and analog-to-digital conversion.

Dr. Börsell is a voting member of the Waveform Measurements and Analysis Technical Committee (TC-10) of the IEEE Instrumentation and Measurement Society.

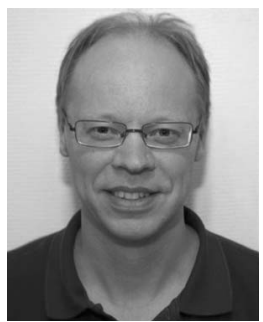

Magnus Jansson (S'93-M'98) received the M.S., Tech. Lic., and Ph.D. degrees from the Royal Institute of Technology (KTH), Stockholm, Sweden, in 1992, 1995, and 1997, respectively, all in electrical engineering

In January 2002, he was appointed Docent in Signal Processing at KTH. Since 1993, he has held various research positions with the Department of Electrical Engineering, $\mathrm{KTH}$, where he is currently an Associate Professor. From 1998 to 1999, he visited the Department of Electrical and Computer Engineering, University of Minnesota, Minneapolis. He is an Associate Editor of EURASIP Journal of Advances in Signal Processing. His research interests include navigation and positioning, sensor array processing, time series analysis, and system identification.

Dr. Jansson is an Associate Editor of the IEEE Signal Processing LETTERS. 